\title{
Qualidade da farinha obtida de grãos de trigo fumigados com dióxido de carbono e fosfina
}

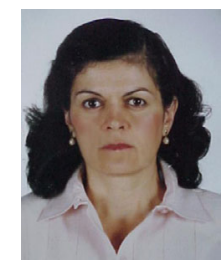

\author{
Lêda R. D. Faroni ${ }^{1}$, Pedro A. Berbert ${ }^{2}$, Ana P. Martinazzo ${ }^{1}$ \& Enilce M. Coelho ${ }^{2}$ \\ 1 DEA/UFV. CEP 36571-000, Viçosa, MG. Fone: (31) 3899-1874. E-mail: Ifaroni@ufv.br (Foto) \\ 2 UENF/CCTA/LEAG. CEP 28015-620, Campos, RJ. Fone: (22) 2726-1607. E-mail: pberbert@uenf.br
}

Protocolo $130-14 / 9 / 2001$

\begin{abstract}
Resumo: Avaliar a qualidade da farinha obtida de grãos de trigo fumigados com atmosfera sintética $\left(21 \%\right.$ de $\left.\mathrm{CO}_{2}\right)$ associada a níveis reduzidos de fosfina $\left(0,25,0,50\right.$ e $\left.0,75 \mathrm{~g} \mathrm{~m}^{-3}\right)$ foi o que se objetivou através deste trabalho. Para posterior comparação dos resultados, realizaram-se dois tratamentos com ar ambiente associado às dosagens de zero e $1,0 \mathrm{~g} \mathrm{~m}^{-3} \mathrm{PH}_{3}$, em três períodos de exposição $\left(24,72\right.$ e 120 h) e temperatura de $29^{\circ} \mathrm{C}$ e $60 \%$ de umidade relativa. A análise dos resultados permitiu concluir-se que, em geral, os tratamentos não influenciaram na qualidade da farinha para panificação obtida do trigo fumigado com fosfina associada à atmosfera sintética $\left(21 \%\right.$ de $\left.\mathrm{CO}_{2}\right)$ e ao ar ambiente.
\end{abstract}

Palavras-chave: armazenamento de grãos, atmosfera controlada, trigo

\section{Quality of flour obtained from wheat grains fumigated with carbon dioxide and phosphine}

\begin{abstract}
The objective of present study was to evaluate the quality of wheat flour dough as affected by fumigation with a synthetic atmosphere containing $21 \% \mathrm{CO}_{2}$ associated with low levels of phosphine $\left(0.25,0.50\right.$ and $\left.0.75 \mathrm{~g} \mathrm{~m}^{-3}\right)$. For comparison purposes, two tests were carried out using atmospheric air associated with 0 and $1.0 \mathrm{~g} \mathrm{~m}^{-3}$ of phosphine. All treatments were submitted to three exposure times $\left(24,72\right.$ and $120 \mathrm{~h}$ ), at $29^{\circ} \mathrm{C}$ and $60 \%$ relative humidity. Results indicated that none of the treatments had any deleterious effects on wheat flour dough for bread making.
\end{abstract}

Key words: grain storage, controlled atmosphere, wheat

\section{INTRODUÇÃO}

O controle de insetos em grãos armazenados é um dos aspectos mais relevantes na etapa de pré-processamento de produtos agrícolas. Por ser efetivo, de baixo custo e de fácil manejo, o controle químico tradicional tem sido a forma mais utilizada em todo o mundo para a proteção de grãos armazenados contra a infestação de insetos, cuja crescente resistência aos agentes químicos, a possibilidade de intoxicação dos operadores e a presença de resíduos nos alimentos, levaram à busca de alternativas menos danosas ao homem e que proporcionassem menor impacto ambiental. Uma alternativa encontrada é a proteção do produto armazenado por meio de atmosferas modificadas.

Martinazzo et al. (2000) avaliaram o efeito de fosfina associada a atmosferas ricas em dióxido de carbono no controle efetivo do inseto $R$. dominica, em todas as suas fases de desenvolvimento. Realizaram-se fumigações com atmosfera sintética $\left(21 \%\right.$ de $\left.\mathrm{CO}_{2}\right)$, associada a níveis reduzidos de fosfina $\left(0,25,0,50\right.$ e $\left.0,75 \mathrm{~g} \mathrm{~m}^{-3}\right)$. Para posterior comparação dos resultados, foram realizados dois tratamentos com ar ambiente associado às dosagens de zero e $1,0 \mathrm{~g} \mathrm{~m}^{-3} \mathrm{PH}_{3}$. Todos os tratamentos se deram em três períodos de exposição $(24,72$, e $120 \mathrm{~h}$ ), na temperatura de $29^{\circ} \mathrm{C}$ e com $60 \%$ de umidade relativa. A análise dos resultados do controle dos insetos permitiu concluir-se que o aumento no período de exposição resultou no aumento da eficácia dos tratamentos em atmosferas sintética e ambiente. Em geral, o controle efetivo de todas as fases do inseto foi obtido com $0,50 \mathrm{e} 0,75 \mathrm{~g} \mathrm{~m}^{-3} \mathrm{PH}_{3}+\mathrm{CO}_{2} \mathrm{em} 120 \mathrm{~h}$. Ovos e adultos foram as fases mais susceptíveis, e a de pupa, a mais resistente, porém não existem informações disponíveis na literatura sobre o efeito da fumigação com fosfina, associada a atmosferas ricas em dióxido de carbono, na qualidade da farinha obtida de grãos de trigo submetidos a esse tratamento. Na indústria de alimentos, a qualidade do trigo é julgada por sua conveniência para determinado tipo de uso. Embora a variedade seja fator influente na qualidade do trigo, este é raramente posto no mercado, baseando-se apenas numa variedade individual. É prática comum separar o trigo, na comercialização, de acordo com a classe, em que cada classe consiste em um 
grupo de variedades com características de certa forma semelhantes e geralmente usadas para propósitos similares. Como acontece com os grãos de trigo, a qualidade da farinha varia diferentemente para usos distintos nos produtos, além de ser definida pela sua capacidade de produzir, uniformemente, um produto final atrativo e com custo competitivo e ainda, ser representada, usualmente, por várias medidas e características que as experiências têm indicado como significantes no uso final.

Um dos principais testes para avaliação da qualidade da farinha de trigo é aquele realizado utilizando-se o farinógrafo. O farinógrafo de Brabender tem tido ampla aceitação nos laboratórios de cereais para controlar as propriedades de mistura das massas de farinha de trigo, e se destina a avaliar a qualidade de uma farinha quanto à sua capacidade de absorver água e resistir ao amassamento durante os processos de fabricação de pães e produtos correlatos. Os valores mais comuns para se interpretar o farinograma e, portanto, avaliar as propriedades da farinha, são: absorção de água, tempo de desenvolvimento da massa, estabilidade e índice de tolerância (Shuey, s.d., apud D'Apolinia \& Kunerth, s.d.).

A absorção de água é definida como a quantidade de água necessária para o centro da curva do farinograma alcançar a linha das 500 unidades farinográficas (U.F.) para uma massa farinha-água, fato este considerado fator de importância na produção de todos os tipos de espécies de fornada (Pyler, 1988). Os altos valores de absorção são desejáveis, desde que eles aumentem o rendimento das espécies. As variações no conteúdo de proteína e amido danificado da massa, são conhecidas como os fatores de maior influência na absorção de água (Preston \& Kilborn, s.d., citados por D'Apolinia \& Kunerth, s.d.).

O tempo de desenvolvimento da massa é uma outra indicação da qualidade da proteína; farinhas fortes normalmente requerem um tempo de desenvolvimento maior que farinhas fracas; no farinograma é definido como o tempo desde o início até o desenvolvimento máximo da curva, imediatamente antes da primeira indicação da queda. Este valor é também chamado tempo de pico. Ocasionalmente, dois picos podem ser observados, em que o segundo deve ser tomado como ponto de desenvolvimento da massa (Pyler, 1988).

A estabilidade é um índice primário da qualidade da farinha e uma das mais significativas determinações realizadas pelo farinógrafo. É definida como a diferença entre o tempo relativo ao ponto em que o topo da curva intercepta a linha das 500 U.F. e o tempo relativo ao ponto em que o topo da curva deixa a linha das 500 U.F. Em geral, este valor dá alguma indicação da tolerância à mistura que a farinha terá (Shuey, s.d., citado por D'Apolinia \& Kunerth, s.d.).

Outro parâmetro qualitativo a ser determinado é o Índice de Tolerância à Mistura (M.T.I.). No farinograma, o valor do M.T.I. é a diferença, em unidades de Brabender, entre o total da curva no pico e o topo da curva medida $5 \mathrm{~min}$ depois do pico ser alcan-çado. Comumente, as farinhas que têm boa tolerância à mistura têm menor M.T.I. Quanto maior for o índice de tolerância, mais fraca é a farinha (Shuey, s.d., citado por D'Apolinia \& Kunerth, s.d.).

Outros testes para avaliação da qualidade da farinha de trigo são aqueles realizados utilizando-se o extensógrafo. Este aparelho se destina a avaliar as características da massa, como a elasticidade e a extensibilidade e, conseqüentemente, a sua capacidade de retenção de gás durante o processo de fermentação. A elasticidade ou resistência à extensão é a propriedade que relaciona a extensão à força aplicada na massa. Quando cessa a ação da força, a massa volta ao seu estado original. No extensograma, é o valor, em unidades de Brabender, obtido no ponto mais alto da curva a $50 \mathrm{~mm}$ depois do início da curva (Pyler, 1988). A extensibilidade é a propriedade da massa de poder se estender, não recuperando o estado inicial. No extensograma, é o comprimento em $\mathrm{mm}$ desde o início até o fim da curva. A extensibilidade corresponde à extensão da massa equivalente ao seu comprimento original (Pyler, 1988).

Além dos parâmetros descritos acima, há que se determinar, ainda, o número proporcional, ou seja, a relação entre a resistência à extensão e a extensibilidade (R/EX). Esse número indica a força, em unidades extensográficas, necessária para esticar a massa em $1 \mathrm{~mm}$ (Pyler, 1988). Este valor dá uma idéia do comportamento de massa: se o número proporcional for pequeno, maior será a tendência da massa fluir e vice-versa, isto é, quanto maior o número proporcional, maior a tendência da massa encolher.

Finalmente, determina-se, também, o índice de queda ("falling number") o qual representa a medida da viscosidade de uma pasta de farinha adicionada de água aquecida em banhomaria, de forma a demonstrar a atividade da alfa-amilase na amostra. A atividade da enzima é expressa em termos de "falling number”, que é o tempo, em segundos, necessário para mover uma suspensão de farinha e permitir que um "viscosimeterstirrer" atravesse uma distância afixada através de uma suspensão quente aquosa de farinha, liquefeita pela enzima em um aparelho padronizado (Pyler, 1988). Quanto mais viscosa a pasta, maior é o índice de queda e menor é a atividade da alfaamilase. O primeiro efeito causado pela hidrólise da enzima alfa-amilase é a diminuição da viscosidade devido a quebras no interior da molécula de amido. A alfa-amilase tem várias funções na produção do pão e a mais importante é a liberação de açúcares do amido durante a fermentação. Esses açúcares servem de substrato para as leveduras que produzem gás carbônico.

De acordo com o exposto e tendo em vista a escassez de dados na literatura, este trabalho objetivou avaliar a qualidade da farinha obtida de grãos de trigo fumigados com dióxido de carbono e fosfina, nas condições estudadas por Martinazzo et al. (2000).

\section{MATERIAL E MÉTODOS}

O presente trabalho foi realizado no Setor de PréProcessamento de Produtos Agrícolas do Departamento de Engenharia Agrícola, na Universidade Federal de Viçosa, MG. Para avaliação do efeito de atmosferas modificadas, associadas a níveis reduzidos de fosfina, na qualidade da farinha produzida a partir de grãos de trigo não-infestados, utilizou-se um lote de grãos fornecido pelo Moinho Vera Cruz, localizado em Santa Luzia, MG. O produto era proveniente de uma mistura de diversas variedades de trigo, cuja moagem resulta em farinha destinada à panificação. Para se determinar se os gases 
utilizados na desinfestação dos grãos tiveram algum efeito sobre a qualidade da farinha do trigo fumigado, realizaram-se análises de farinografia, extensografia e índice de queda, no laboratório do próprio moinho.

A dosagem mínima de fosfina recomendada pelos fabricantes para o controle efetivo de insetos em grãos armazenados, é de $1,0 \mathrm{~g} \mathrm{~m}^{-3}$ (Compendio..., 1996). Sendo assim, este experimento foi realizado utilizando-se três dosagens de fosfina $\left(0,25,0,50\right.$ e $\left.0,75 \mathrm{~g} \mathrm{~m}^{-3}\right)$ associadas a uma atmosfera sintética contendo $79 \%$ de $\mathrm{N}_{2}, 21 \%$ de $\mathrm{CO}_{2}$ e $0 \%$ de $\mathrm{O}_{2}$. Para a comparação dos resultados, as amostras foram também submetidas a dois tratamentos com ar atmosférico $\left(79 \%\right.$ de $\mathrm{N}_{2}$, $0,03 \%$ de $\mathrm{CO}_{2}$ e $21 \%$ de $\mathrm{O}_{2}$ ) utilizando-se as dosagens de zero e $1,0 \mathrm{~g} \mathrm{~m}^{-3} \mathrm{PH}_{3}$. Em todos os tratamentos, a fosfina foi obtida a partir da reação do fosfeto de alumínio (AlP) em forma de pastilha, com a água contida no ar atmosférico, de acordo com a reação química: $\mathrm{AlP}+3 \mathrm{H}_{2} \mathrm{O} \rightarrow \mathrm{Al}(\mathrm{OH})_{3}+\mathrm{PH}_{3}$.

Foram analisados três períodos de exposição $(24,72$ e 120 h) e apenas um nível de temperatura, $29^{\circ} \mathrm{C}$. O delineamento experimental foi inteiramente casualizado, composto de 13 tratamentos, com três repetições para cada tratamento, num total de 39 parcelas. Esse procedimento visava à identificação das condições ideais de controle dos insetos com a atmosfera modificada, tomando-se como base o controle obtido com a dosagem recomendada $\left(1,0 \mathrm{~g} \mathrm{~m}^{-3}\right) \mathrm{em}$ ar ambiente.

A determinação da qualidade da farinha pelo farinógrafo foi realizada de acordo com a metodologia prescrita pela American Association of Cereal Chemists (AACC, 1996) que exige que as amostras de farinha sejam analisadas em dois estágios; no primeiro, chamado curva de titulação, pesavam-se $300 \mathrm{~g}$ de farinha em função do teor de umidade. Com o farinógrafo em movimento adicionava-se, desde a bureta, uma quantidade de água, até que aparecesse uma linha contínua no diagrama que indicasse 500 unidades farinográficas. A quantidade de água adicionada em porcentagem representava a absorção de água dessa farinha e, no segundo estágio, foi traçado o farinograma propriamente, para o qual se pesava novamente a farinha e se adicionava à quantidade de água determinada previamente, deixando-se o aparelho em movimento, pelo período de $20 \mathrm{~min}$.

A determinação da qualidade da farinha pelo extensógrafo também foi realizada de acordo com a metodologia prescrita pela AACC (1996). As massas testadas no extensógrafo eram preparadas no farinógrafo, com uma consistência de 500 U.F. e com um tempo de mistura apropriado, adicionando-se $6 \mathrm{~g}$ de sal de cozinha $(\mathrm{NaCl})$. Depois de preparada no farinógrafo, pesavam-se duas massas de $150 \mathrm{~g}$ cada. A massa de teste era boleada e modelada, posteriormente, em dispositivos especiais do extensógrafo. Logo a seguir, as massas eram colocadas na cabine de fermentação do extensógrafo e, depois de $45 \mathrm{~min}$, a massa que ficou fixada por fixadores especiais era colocada no suporte do aparelho e esticada pelo gancho, até quebrar. A força requerida para esticar a massa era transmitida por um sistema calibrado até o registrador, que grava a curva num papel apropriado. Depois de a mesma massa ser boleada e moldada, novamente se repetia a operação duas vezes. O tempo total de fermentação é de $135 \mathrm{~min}$, sendo obtidas três curvas que representam os tempos de fermentação de 45, 90 e 135 min. As mudanças observadas na forma das curvas, aos $135 \mathrm{~min}$, proporcionam alguma indicação de tolerância à fermentação da farinha, assim como os tempos relativos de fermentação.

Os valores do índice de queda foram determinados por um aparelho "falling number", onde $7 \mathrm{~g}$ de farinha foram colocados em um tubo com $25 \mathrm{~mL}$ de água destilada, preso por uma haste ao aparelho e agitado em banho-maria $\left(100^{\circ} \mathrm{C}\right)$ por $60 \mathrm{~s}$, para se obter uma suspensão uniforme; em seguida, o movimento era automaticamente interrompido e era determinado o tempo, em segundos, que a haste do aparelho levava para passar pela suspensão. A análise da qualidade da farinha obtida dos grãos de trigo foi realizada utilizando-se a estatística descritiva.

\section{RESULTADOS E DISCUSSÃO}

Para a avaliação da qualidade da farinha obtida dos grãos de trigo fumigados com atmosfera sintética $\left(21 \%\right.$ de $\left.\mathrm{CO}_{2}\right)$ e 0,25 , $0,50 \mathrm{e} 0,75 \mathrm{~g} \mathrm{~m}^{-3}$ de fosfina e atmosfera ambiente com $1,0 \mathrm{~g} \mathrm{~m}^{-3} \mathrm{de}$ $\mathrm{PH}_{3}$, em três períodos de exposição, 24,72 e $120 \mathrm{~h}$, foram realizadas análises pelo farinógrafo e extensógrafo e, finalmente, análise do índice de queda. Os resultados foram comparados com os sugeridos pela classificação de Preston \& Kibborn (s.d.) citados por D'Apolinia \& Kunerth (s.d.) - Tabela 1, para posterior classificação do trigo fumigado. Uma amostra de trigo não-tratada

Tabela 1. Classificação da farinha de trigo sugerida por Preston \& Kibborn (s.d.)

\begin{tabular}{lcccc}
\hline \multirow{2}{*}{ Característica } & \multicolumn{4}{c}{ Trigo } \\
\cline { 2 - 5 } & $\mathrm{F}^{1}$ & $\mathrm{M}^{2}$ & $\mathrm{Ft}^{3}$ & $\mathrm{E} \mathrm{Ft}^{4}$ \\
\hline Desenvolvimento (min) & $<2,5$ & $2,5-4,0$ & $4,0-8,0$ & $>10$ \\
M.T.I. (UB) & $>100$ & $60-100$ & $15-50$ & $<10$ \\
Absorção (\%) & $<55$ & $54-60$ & $>58$ & \\
Proteína (\%) & $7,5-9,0$ & $10-11,5$ & $>11,5$ \\
Amido danificado (uF) & $0-10$ & $10-20$ & $15-30$ & 15 \\
\hline 'F - Fraco: produtos para pastelaria, crackers, talharim \\
2 M - Médio: crackers, talharim, pães de volume pequeno \\
${ }^{3}$ Ft - Forte: utilizada como mistura para aumentar a resistência de farinhas mais fracas \\
${ }^{4}$ E Ft - Extra Forte: utilizadas em mistura com outras farinhas \\
${ }^{5}$ M.T.I. (UB) - Mixing Tolerance Index (Unidades de Brabender)
\end{tabular}

foi também analisada para fins de comparação.

Na Tabela 2, apresentam-se os valores médios obtidos na análise da farinha resultante dos grãos de trigo não-fumigados. Comparando-se esses valores com os apresentados na Tabela 1 de Preston \& Kibborn, citados por D'Apolinia \& Kunerth (s.d.), o trigo deveria ser considerado "médio"; no entanto, a moagem realizada em laboratório é feita em um moinho experimental, o que não resulta em uma boa granulometria e, conseqüentemente, obtém-se menor quantidade de amido danificado, um dos fatores responsáveis pela maior absorção de água pela farinha. Quando a moagem é realizada em grande escala, ou seja, de forma semelhante ao que se faz em indústrias Tabela 2. Valores médios obtidos na análise de qualidade da farinha de trigo não-tratado

\begin{tabular}{lclc}
\hline \multicolumn{1}{c}{ Farinografia } & $\begin{array}{c}\text { Amostra } \\
\text { Inicial }\end{array}$ & Extensografia & $\begin{array}{c}\text { Amostra } \\
\text { Inicial }\end{array}$ \\
\hline Absorção (\%) & 56,6 & Resistência (UB) & 400 \\
Desenvolvimento (min) & 13,0 & Extensibilidade (mm) & 170,0 \\
Estabilidade (min) & 15,5 & Resist./extensib. & 2,4 \\
M.T.I. (UB) & 40,0 & Índice de queda & - \\
\hline "resist./extensib. - resistência/extensibilidade & &
\end{tabular}


moageiras, os valores de absorção aumentam cerca de 2 a 3 pontos percentuais. Por esta razão, o trigo que não recebeu nenhum tratamento foi classificado como "forte".

Os valores médios obtidos nas análises das farinhas resultantes dos grãos de trigo fumigados com fosfina em atmosfera sintética $\left(21 \%\right.$ de $\left.\mathrm{CO}_{2}\right)$ e em atmosfera ambiente, nos períodos de exposição de 24, 72 e 120 h, está apresentado na Tabelas 3.

Tabela 3. Valores médios obtidos na análise da qualidade da farinha resultante dos grãos de trigo fumigados com atmosfera sintética $\left(21 \%\right.$ de $\left.\mathrm{CO}_{2}\right)$ e $0,25,0,50$ e $0,75 \mathrm{~g} \mathrm{~m}^{-3} \mathrm{PH}_{3}$ e atmosfera ambiente, com 1,0 $\mathrm{g} \mathrm{m}^{-3}$ de fosfina, no período de exposição de 24 (A), 72 (B) e 120 h (C)

\begin{tabular}{|c|c|c|c|c|}
\hline Farinografia & $\begin{array}{c}21 \% \\
\mathrm{CO}_{2}+ \\
0,25 \\
\mathrm{~g} \mathrm{~m}^{-3} \mathrm{PH}_{3}\end{array}$ & $\begin{array}{c}21 \% \\
\mathrm{CO}_{2}+ \\
0,5 \\
\mathrm{~g} \mathrm{~m}^{-3} \mathrm{PH}_{3}\end{array}$ & $\begin{array}{c}21 \% \\
\mathrm{CO}_{2}+ \\
0,75 \\
\mathrm{~g} \mathrm{~m}^{-3} \mathrm{PH}_{3}\end{array}$ & $\begin{array}{c}\text { Ambiente } \\
1,00 \\
\mathrm{~g} \mathrm{~m}^{-3} \mathrm{PH}_{3}\end{array}$ \\
\hline \multicolumn{5}{|c|}{ A. Período de Exposição de $24 \mathrm{~h}$} \\
\hline Absorção (\%) & 56,75 & 55,25 & 56,47 & 57,50 \\
\hline Desenv. (min) & 11,25 & 10,50 & 11,0 & 10,50 \\
\hline Estabilidade (min) & 15,75 & 13,50 & 14,33 & 14,00 \\
\hline M.T.I. (UB) & 45,0 & 35,00 & 36,67 & 40,00 \\
\hline \multicolumn{5}{|l|}{ Extensografia } \\
\hline Resistência (UB) & 380,0 & 385,00 & 350,0 & 346,67 \\
\hline Extensibilidade (mm) & 161,67 & 157,50 & 163,33 & 160,00 \\
\hline Resist./Extensib. & 2,37 & 2,45 & 2,2 & 2,20 \\
\hline Índice de queda & 330,0 & 321,00 & 301,67 & 302,33 \\
\hline \multicolumn{5}{|c|}{ B. Período de Exposição de $72 \mathrm{~h}$} \\
\hline Absorção (\%) & 56,93 & 56,13 & 56,30 & 55,93 \\
\hline Desenv. (min) & 7,50 & 10,67 & 12,25 & 13,33 \\
\hline Estabilidade (min) & 13,67 & 15,67 & 14,25 & 15,50 \\
\hline M.T.I. (UB) & 43,33 & 40,00 & 30,0 & 40,00 \\
\hline \multicolumn{5}{|l|}{ Extensografia } \\
\hline Resistência (UB) & 353,33 & 390,00 & 345,0 & 450,00 \\
\hline Extensibilidade (mm) & 178,33 & 151,67 & 167,5 & 146,67 \\
\hline Resist./Extensib. & 2,35 & 2,60 & 2,05 & 3,17 \\
\hline Índice de queda & - & - & 335,0 & 321,67 \\
\hline \multicolumn{5}{|c|}{ C. Período de Exposição de 120 h } \\
\hline Absorção (\%) & 56,47 & 56,67 & - & 57,27 \\
\hline Desenv. (min) & 7,67 & 12,50 & - & 8,33 \\
\hline Estabilidade (min) & 14,33 & 16,33 & - & 15,50 \\
\hline M.T.I. (UB) & 36,67 & 30,00 & - & 40,00 \\
\hline Extensografia & & & - & \\
\hline Resistência (UB) & 350,00 & 420,00 & - & 376,67 \\
\hline Extensibilidade (mm) & 163,33 & 161,67 & - & 161,67 \\
\hline Resist./extensib. & 2,20 & 2,60 & - & 2,33 \\
\hline Índice de queda & - & 56,67 & - & - \\
\hline
\end{tabular}

Comparando-se os valores de absorção apresentados na Tabela 3 (A, B e C) com os da tabela de classificação sugerida por Preston e Kibborn (s.d.) citados por D’Apolinia \& Kunerth (s.d.), verifica-se que a classificação do trigo depois de fumigado com fosfina em atmosfera sintética $\left(21 \% \mathrm{CO}_{2}\right)$ e em atmosfera ambiente, manteve-se como "forte".

Quanto ao tempo de desenvolvimento da massa, embora o trigo não-tratado (Tabela 2), e alguns fumigados tenham apresentado valores superiores a $10 \mathrm{~min}$ (Tabela 3A, B e C), os quais levariam à classificação de "extra-forte", Pyler (1988) sugere que farinhas "extra-fortes" necessitam de uma velocidade de mistura no farinógrafo de 90 rotações min $^{-1}$. Como nas análises realizadas com o trigo dos diversos tratamentos foi aplicada uma velocidade de 62 rotações min $^{-1}$, não se justifica sua classificação como “extra-forte”. Sendo assim, em relação ao tempo de desenvolvimento, tanto a farinha obtida de grãos de trigo não-fumigados quanto aquela obtida de grãos fumigados com fosfina em atmosfera sintética são classificadas como "forte".

\section{CONCLUSÕES}

1. A farinha obtida de trigo não-tratado e inicialmente classificada como "forte", manteve essa classificação quando produzida tanto a partir de grãos de trigo fumigados com atmosfera sintética $\left(21 \%\right.$ de $\left.\mathrm{CO}_{2}\right)$ e fosfina $\left(0,25,0,50\right.$ e $\left.0,75 \mathrm{~g} \mathrm{~m}^{-3}\right)$ quanto em atmosfera ambiente, com $1,0 \mathrm{~g} \mathrm{~m}^{-3} \mathrm{de} \mathrm{PH}_{3}$, até períodos de exposição de $120 \mathrm{~h}$.

2. Não houve grande variação nos valores médios de estabilidade de cada tratamento, quando se compara a farinha obtida a partir de grãos não-tratados de trigo com aquelas produzidas utilizando-se grãos de trigo fumigados com fosfina em atmosfera sintética ( $21 \%$ de $\left.\mathrm{CO}_{2}\right)$ e em atmosfera ambiente, nos três períodos de exposição.

\section{LITERATURA CITADA}

AAAC - American Association of Cereal Chemists. Approved methods of the AACC, 9th ed. Method 56-81B. 1995.

Compêndio de defensivos agrícolas; guia prático de produtos fitosanitários para uso agrícola. 5.ed. São Paulo: Organização Andrei, 1996. 250p.

D’Apolinia B. L.; Kunerth, W. H. The farinograph handbook. 3.ed. [S.1.: s.d.], 63p.

Gastoxin, Manual técnico (fosfeto de alumínio). São Vicente: Casa Bernardo. s.d. 28p.

Martinazzo, A.P.; Faroni, L.R.D.; Berbert, P.A.; Reis, F.P. Utilização da fosfina em combinação com o dióxido de carbono no controle do Rhyzopertha dominica (F.). Pesquisa Agropecuária Brasileira, Brasília, v.35, n.6, p.1063-1069, 2000.

Pyler, E. J. Baking - Science \& Tecnology. 3.ed. vol II. Kansas: Sosland Publishing Company. 1988. 